\title{
Cross-cultural validation of the Health Care Provider HIV/AIDS Stigma Scale (HPASS) in China
}

Hong Xie1, Huiting Yu2, Roger Watson3, Jing Wen4, Lu Xiao5, MaoYan6, Yanhua Chen7*

1. School of Nursing, Southwestern Medical University, Luzhou, China

2. School of Nursing, Southwestern Medical University, Luzhou, China

3. Health and Social Care Faculty, the University of Hull, UK

4. School of Nursing, Southwestern Medical University, Luzhou, China

5.Respiratory Medicine Department of the Affiliated Hospital of Southwestern Medical University, Luzhou, China

6. Infectious Disease Department of the Affiliated Hospital of Southwestern Medical University, Luzhou, China

7. Infectious Disease Department of the Affiliated Hospital of Southwestern Medical University, Luzhou, China

*Corresponding author: Yanhua Chen

Address: Infectious Disease Department of the Affiliated Hospital of Southwestern Medical University, 25, Taiping Street, Luzhou, China

Phone: +8608302792129

Email: chen_yanhua25@163.com

Fax: 008608303160244

\section{Acknowledgements}


The authors thank all the participants. We acknowledge the editors and the reviewers for insightful suggestions on this work.

\section{Competing interests}

All the authors do not have any possible conflicts of interest.

\section{Ethical approval}

Ethical approval was granted by the research ethics committee of the Affiliated Hospital of Southwestern Medical University, in China.

\section{Informed consent}

Informed consent was obtained from all individual participants included in the study. 


\section{Cross-cultural validation of the Health Care Provider HIV/AIDS Stigma Scale (HPASS) in China}

1 Abstract

2 Introduction: The study aimed to validate the Health Care Provider HIV/AIDS 3 Stigma Scale (HPASS) among medical staff in China.

4 Methods: The validation was conducted in four steps from March to December 2017:

5 translation and back-translation; content validity test with six experts; test-retest 6 reliability testing with 63 medical staff with two weeks interval; and structural 7 validation with 349 medical staff from 52 hospitals with a convenience sample, using 8 exploratory factor analysis, including principal component analysis and varimax 9 rotation.

Results: The Scale Content Validity Index Average(S-CVI/Ave) was 0.88, while for test-retest reliability, the ICC was 0.87 . Three factors of "discrimination", "prejudice" and "stereotype" with 16 items were extracted and explained 59.61\% variance. The Cronbach's alpha value for the total scale was of 0.88 , and for the three factors, the values were $0.89,0.86$ and 0.74 , respectively. The discrimination factor showed identical means between Canadian medical students and Chinese medical staff, while the prejudice and stereotype factors had higher mean scores in the Chinese sample.

Conclusion: The three-factor structure of Health Care Provider HIV/AIDS Stigma Scale was confirmed in Chinese medical staff with a simpler solution. This could provide a basis for trans-cultural application and comparison.

Keywords: medical staff; HIV/AIDS; stigma; cross-culture validation; scale.

\section{Introduction}

Current stigma and discrimination against people living with HIV (PLWHA) have been recognized as key impediments to controlling the HIV epidemic [1]. Research shows that stigma may deter people living with HIV from actively seeking medical care, and their health may further deteriorate because of discrimination and 
1 prejudice from medical workers [2]. Furthermore, along with the success of

2 anti-retroviral treatment, HIV/AIDS becomes a chronic condition rather than an acute

3 disease; hence medical staff has more opportunities to provide care to PLWHA.

4 Therefore, anti-discrimination, especially elimination of AIDS-related discrimination

5 from medical personnel, is an important part of AIDS prevention and care work.

In China, along with the development of anti-HIV related stigma campaign, institutional discrimination towards PLWHA has diminishing dramatically in the field of health care [3].Medical insurance for PLWHA is ensured, furthermore, arbitrary testing for HIV or notification to partner or relatives, and compulsory internment are prohibited particularly after the issue of the Regulation of AIDS Prevention and Treatment in 2003[4]. But some PLWHA report being refused admission to hospitals by health care workers because of their HIV-positive status [5], which makes the treatment of PLWHA difficult. Frequently AIDS patients must go to the infectious disease hospitals for treatment rather than general hospital, where PLWHA is acceptable for surgical operation, and usually there is only one such hospital in any city[6]. Obviously, combating HIV stigma has not ended.

To understand the intensity of stigma against HIV-positive people by medical staff and assess the effectiveness of anti-stigma intervention, several HIV/AIDS-related stigma measurement scales were developed [7-11]. Some of them were developed before anti-retroviral medicine and some were not theoretically based; in addition, they were used locally. As stigma was culturally diverse and changing with context, this makes the across-culture understanding and comparison problematic.

Some Chinese scales have been developed. Stein and Li [8] developed a scale for medical staff-related stigma, including 17 items and five factors: Discrimination Intent at Work, Prejudiced Attitudes, and Good Care for HIV Patients, Internalized Shame, and Fear of PLWHA. Among the five factors, only two: the Discrimination Intent at Work; and Good Care for HIV Patients assess stigma of medical staff towards AIDS patients, other factors measuring public stigma against PLWHA. The 
1 factor Good Care for HIV Patients evaluates the stigma towards AIDS patients

2 infected HIV through different transmission routes, such as drug abuse, sexual work,

3 and blood donation. However, the epidemic pattern of HIV varies; over 90\% PLWHA

4 are HIV infected by sexual transmission[12]; illegal blood collection has been

5 controlled completely. Alongside the campaign against anti-HIV related stigma, the

6 concept and reality of stigma in health care is changing. Earnshaw's mechanism

7 proposed that internalized shame [13] was mainly about the internal humiliation of

8 AIDS patients, which may not be appropriate to applied to medical staff.

$\mathrm{Tu}$ [14] generated a scale containing: refusing to provide treatment; taking a differential treatment; medical restrictions and isolation; compulsory notification; testing without knowledge; refusing to inform patients of their result; and non-confidentiality, based on the seven kinds of discrimination existing in health care proposed by UNAIDS in 2002[15]. Cao Y et al. [16] composed a scale with 19 items based on the work of Tu [14], including four sub-scales: avoidance; disclosure; stigma; and fear. Currently, phenomena such as compulsory disclosure, medical restriction and isolation, not informing about test results are diminishing, but the prejudice and stereotype towards PLWHA may still prevail. Therefore, these measurements may not detect the severity of real stigma and the effectiveness of anti-stigma intervention on this situation.

Recently the Health Care Provider HIV/AIDS Stigma Scale (HPASS) [7], a psychometrically sound measurement was developed by Wagner, following the scale-up steps recommended by Brown [17], based on the stigma framework of Earnshaw and Chardoier [13], which is a widely accepted model, including three domains: discrimination as behavior response to the infected people; prejudice defined as the emotional attitude operationally; and stereotype as negative belief towards PLWHA. This scale provides a possibility to measure the current stigmatization by health providers. So far, the HPASS scale has only been used in English-speaking countries. Therefore, this study attempted to validate the scale in China and to provide evidence for cross-cultural understanding and comparison. 


\section{Method}

2

\section{Design}

The study was divided into four stages (Figure 1) and a cross-sectional survey was used from March to December 2017.

\section{Research process}

Stage 1: After obtaining consent from the original author of the measurement, two English lecturers in a medical University were invited to translate the scale into Chinese and other two lectures translated the scale back into English. One United Kingdom nursing expert, RW, checked the original and translated version and no change was needed. A bilingual HIV expert conducted cross-cultural adjustment without violating the meaning of the original scale.

Stage 2: Six experts reviewed the contents of the scale and the relevance to stigma. The expert panel included two psychiatrists and four HIV specialists with over 10 years' experience of working with PLWHA, two had PhDs, three had master degree and one a bachelor degree. The relevance of items was rated at four levels, labeled as irrelevant, somewhat relevant, relevant and highly relevant and rated as 1 to 4 points respectively [18].

Stage 3: This stage involved investigating test-retest reliability. In June 2017, five departments of a medical university hospital in Sichuan Province of China were selected. Inclusion criteria of the participants were: 1. Medical staff working in the hospital; 2. Voluntary participation in the study. Exclusion criteria were: nursing or medical students. One hundred and forty questionnaires were collected via Questionnaire Star, a Chinese online survey tool; after 10 days, 64 questionnaires for retesting were collected via the same online survey tool. The response rate was $45.71 \%$ and 63 were valid.

Stage 4: A cross-sectional study was used to explore the construct validity from July to November 2017. The sample size was calculated based on factor analysis requirement, i.e. over 10 times the number of items in the scale [19] and expanded by 
$120 \%$ to account for attrition. Convenience sampling was chosen. The medical staff

2 involved in the survey was from 52 hospitals in Sichuan Province and those

3 participated in stage three were excluded. The data were also collected via

4 Questionnaire Star.

$5 \quad$ Psychometrics

The process of psychometrics establishing is continuous; particularly, building construct validity is a continuous process [20]. Based on Sousa's guideline for cross-cultural validation of a scale [21], this study tested most of psychometrics, e.g. content validity, structural validity, test-retest reliability, internal consistency reliability. Criterion validity was not established as currently no HIV-related stigma scale is recognized as a gold standard. Convergent and divergent validity testing were not conducted due to the limited time and resource of this study.

\section{Measurements}

The questionnaire consisted of three parts, which were used in stages 3 and 4 . The first part was related to the basic attributes of the respondents, including gender, job title, department, etc.; the second part had eight questions about AIDS-related knowledge, with a total score of 8 points. The third part was the HPASS scale. The scale included 30 items, of which Item 15 were reverse scored. The answers were rated from 1 to 6 , from "strongly disagree" to "strongly agree".

\section{Data collection method}

In stages 3 and 4 Questionnaire Star was used to collect data. The URL link to the survey was distributed by the head nurses to the units' member. This questionnaire could only be accessed through the website and could not be retrieved by search engine; each device (mobile phone, computer) or each account could only be used once.

\section{Data analysis}

We used SPSS 23.0 statistical package. The content validation used the Content 
Validity Index (CVI). The criterion of the Item Content Validity Index (I-CVI) was set

2 at 0.78 [22]. The test-retest reliability was measured using Intra-class Correlation

3 Coefficient (ICC) [23], and the two-way random model of absolute agreement type was used. The number of factors was set at three according to the structure of the 5 original scale [7]. Principal components analysis and varimax rotation were applied.

6 The items that met the following conditions were deleted one by one: the factor

loading was less than 0.60 ; the cross loading was over 0.60 ; the absolute value of the

difference between cross loading was over 0.20 .

Ethics consideration

Ethical approval was granted by the research ethics committee of the Affiliated Hospital of Southwestern Medical University, in China. An Informed consent statement was provided to online survey participants and face-to-face respondents, which clearly outlined the rights to confidentiality of their data and their right to withdraw from the study.

\section{Results}

\section{Descriptive characteristics}

The data collection at stage 4 was carried out from September to November 2017. The total number of online surveys was 1017; 364 questionnaires were collected; the response rate was $35.79 \%$; 15 invalid questionnaires were excluded. Therefore, 349questionnaires were included in the data analysis at Stage 4. Gender and age, respondents with different attributes were analyzed in subgroups (Table 1).

\section{Content validity}

The SCVI is defined as: "the proportion of total items judged content valid" or "the proportion of items on an instrument that achieved a rating of 3 or 4 by the content experts" [24]. There are two methods for calculating it, in the universal agreement approach, the number of items considered relevant by all the judges (or number of items with CVI equal to 1) is divided by the total number of items. In the average approach, the sum of I-CVIs is divided by the total number of items [25].The 
1 Scale-Content Validity Index of Universal Agreement(S-CVI/UA) was 0.44 and the

2 Scale Content Validity Index Average(S-CVI/Ave) was 0.88. The Item Content

3 Validity Indexes (I-CVIs) for this scale ranged 0.33-1.00. Based on the I-CVI values

4 (under 0.78) and expert opinion, the item: "I believe I have the right to refuse to treat

5 HIV+ patients if I am concerned about legal liability" was deleted. No additional

6 items were proposed by the experts.

\section{Test-retest reliability}

The total Intra-class Correlation Coefficient (ICC) was 0.87 and the ICCs of items were between 0.20 and 0.75. According to Landis and Koch [26], 11 items in this scale had better test-retest reliability, 15 items normal, two items lower (Item 1:"I believe most HIV +patients acquired the virus through risky behaviour"; Item 3: "I believe I have the right to refuse to treat HIV + patients for the safety of other patients"), and 1 item poor (Item15: "I would be comfortable working alongside another health care provider who has HIV").

\section{Data sorting}

Some cases had missing data for a few individual items. Missing Values were tested. Of the 29 items on the original scale, missing values were less than $5 \%$ and Little's MCAR test showed complete randomization of data $(\mathrm{v} 2=97.437, \mathrm{df}=83, \mathrm{p}=$ 0.133). To maximize the sample retention rate for subsequent analyses, the missing values for the proposed HPASS project were replaced by the estimation maximization (EM) method [27]. Missing values, ceiling and floor effects were assessed as shown in Table 4.

\section{Exploratory factor analysis}

Kaiser- Meyer-Olkin (KMO) was tested, scored 0.90 and Bartlett's test of sphericity was statistically significant $\left(\chi^{2}=4394.350, \mathrm{df}=406, \mathrm{p}<0.001\right)$, which implied that the data were suitable for factor analysis. Three factors, consisting of 16 items, named discrimination (5 items), prejudice (6 items) and stereotype (5 items), were extracted with a total variance of $59.61 \%$ (Tables $2 \& 4$ ). The correlation between 
1 factors is shown in Table 3. The Cronbach's $\alpha$ of the scale was 0.88 , and for the three

2 factors were $0.89,0.86$ and 0.74 , respectively.

\section{HPASS scale score}

There were 16 items in the HPASS scale. The study showed that there was no significant difference in the stigma associated with HIV/AIDS among medical staff ( $p>0.05)$ according to gender, job (doctor and nurse), education level and whether they worked with AIDS. There were significant differences in terms of titles, departments (comparison between Infectious Diseases and Non-Infectious Diseases) and HIV/AIDS-related knowledge scores $(\mathrm{p}<0$.05) (Table 1).

\section{Discussion}

This study examined the psychometrics of the Health Care Provider HIV/AIDS Stigma Scale (HPASS) among medical staff in China, in an across-culture context through four steps. Compared with Wagner's research in Canada, the sample was changed from medical students to medical staff (including doctors, nurses, medical technicians, etc.), but the three factors: "discrimination"; "prejudice"; and "stereotype" were confirmed, with all items loading consistently on these factors, showing that the HPASS scale is understood in the same way between the two countries and has a stable structure across these cultures. The difference was that in this study, to improve the variance of scale interpretation and scale simplicity, 14 items were deleted, and 16 items retained, explaining $59.61 \%$ variance, while the original scale with 30 items explained $48.61 \%$ variance.

Factor 1: "Discrimination" had five questions, including: "I believe I have the right to refuse to treat $\mathrm{HIV}+$ patients if other staff members are concerned about safety" etc. This reflects the medical staff's behavior towards PLWHA. In one study [28], a prevalence rate of HIV/AIDS-related stigma of $15.4 \%$ among the health personnel was observed suggesting that nurses were more likely to give differential care to patients based on their HIV status. The practice of stigma and discrimination of health-care workers against HIV-infected persons also occurs in medical care in 
UK and elsewhere [29]. Stringer et al. [30] found that non-discrimination policy is a useful way to reduce the stigma associated with health-care workers.

Factor 2: "Prejudice" contained six questions, including concern, fear and sense of uncomfortable when health providers treated HIV-positive people. This reflected the medical staff affection and emotion towards HIV/AIDS patients during treatment. Magnus et al. [31] found that the medical staff's attitude played a key role in saving people living with HIV and was crucial to improving and slowing the AIDS epidemic. Although medical staff in China actively gives AIDS patient treatment on the surface, in fact, they have worries and prejudice cognitively in looking after the PLWHA, which indicates intervention to decrease the stigma in this facet is needed. This echoes the theory of Earnshaw, and Chaudoir [13] that the three mechanisms of stigma (discrimination, prejudice and stereotype) are separate processes, and result in varied outcomes. The negative feeling of medical staff towards PLWHA may prevent them from making close relationship with PLWHA, but, nevertheless, as a health provider they would treat PLWHA to meet the requirement of professionalism and certain policies.

Factor 3: "Stereotypes" included five questions. It contained the medical staff's belief that the cause of HIV infection was due to the infected person being unclean, irresponsible, immoral or illegal. The steep incensement of stigma in this factor shows the difference between the two samples and the difference between behavior, affect and belief. Policies, professionalism and ethics have much more powerful impact over the behavior of health providers while less influence on affect and belief, and more effective methods need to be introduced to change the situation. This also implies a hierarchical tendency of stigma in this study, and more precise statistics such as Mokken scale analysis could be used to test this [32].

During the expert panel review, the item: "I believe I have the right to refuse to treat HIV+ patients if I am concerned about legal liability" scored CVI of 0.40 and was deleted. This item is culturally incompatible. In China, to protect the rights of PLWHA, the law of communicable disease prevention and treatment and regulations 
of AIDS was issued, in which the stigma towards PLWHA is prohibited, therefore for legal concern, medical staff should not refuse to treat HIV+ patients.

The internal consistency coefficient of the total score of the scale was 0.88 , which was a little lower than the Canadian study [7], this mainly due to 14 items being deleted, as internal consistency is related to the length of a scale. All the sub-scales' Cronbach's a ranged from 0.74-0.86, indicating good internal consistency.

The overall test-retest reliability was good (ICC $=0.87$ ), indicating that the scale had stability across time, while the original paper did not report ICC value of its test-retests reliability, which made the comparison impossible. Item 15: "I would be comfortable working alongside another health care provider who has HIV." scored ICC of 0.10 was dropped. This may be due to the reverse scoring as stated by Streiner and Norman [20].

If more than $15 \%$ of subjects eventually fall within the lowest or highest score, there is a floor or ceiling effect [33]. Three items in the scale showed different degrees of floor effect, and one item manifested ceiling effect (Table 4). Although there were six levels of options, the ceiling and the floor effect coexisted, which reduced the reliability of the measurement and indicated more options are needed, possibly seven. It may also be influenced by the sample.

This study showed that the stigma scores were not significantly different $(\mathrm{p}>0.05)$ in terms of gender, job (doctor and nurse), education level and whether have worked with AIDS. This contrasted with the findings of Famoroti et al. [5]. Their research showed that there was association between level of education, occupation, gender, and knowledge of HIV/AIDS. The results of this study may be related to the uneven numbers of respondents. The difference was statistically significant $(\mathrm{p}<0.05)$ in terms of the title of medical staff, departments (Infectious Diseases and Non-Infectious Diseases). Staff with senior title(consultant doctor or consultant nurse)scored lower than those with intermediate(charge doctor or charge nurse)and primary title (resident doctor or resident nurse or primary nurse), which may attribute to their clinical experience. From the department's point view, infectious diseases medical staff's 
1 attitude towards AIDS patients is better than other departments, which may relate to

2 their frequently contact with AIDS. This is the same as Doka showed in that older

3 health carers reported less discriminatory attitudes at work than their younger

4 counterparts [28].

\section{$5 \quad$ Research limitations and recommendations}

This study validated the HPASS in China and the scale manifested across culture measurement invariance primarily, i.e. configural invariance (same number of factors and same items (16 remained items) loading on the same factors across culture groups), but more advanced and precise measurement invariance tests are required, such as multiple group invariance test in CFA and Rasch could be applied to find out the measurement invariance from other three levels: metric invariance (the loading of factors are same across groups), scalar invariance (item intercept are consistent) and invariance of measurement error. Furthermore, for across culture comparison, data sharing or research cooperation internationally are also required.

This study found the difference in three factors of stigma among medical staff: the behavior factor showed lowest stigma, while the affection and belief domain showed more severe stigma. The cause of the differences and the effective strategies to resolve this question needs further study. The respondents were recruited from Sichuan province, so the generalizability was limited.

\section{Conclusion}

To sum up, the HPASS scale was validated in across-cultural context with four steps and showed good content validity, stability, internal consistency and a consistent structure with the original study, i.e. discrimination, prejudice and stereotype. The Chinese HIV/AIDS stigma scale could be used to evaluate the stigma associated with HIV/AIDS patients among Chinese medical staff and provide the basis for a cross-cultural comparison.

\section{References}

[1] Srithanaviboonchai, K., Stockton, M., Pudpong, N., et al. Building the evidence base for stigma and discrimination-reduction programming in Thailand: development 
1 of tools to measure healthcare stigma and discrimination. BMC Public Health, 2017,

$2 \quad 17(1): 245$.

3 [2]Famoroti T O, Fernandes L, Chima S C. Stigmatization of people living with

4 HIV/AIDS by healthcare workers at a tertiary hospital in KwaZulu-Natal, South

5 Africa: a cross-sectional descriptive study. Bmc Medical Ethics, 2013, 14(Suppl

$6 \quad$ 1):S6-S6.

7 [3] Wu S, Li L, Wu Z, et al. A brief HIV stigma reduction intervention for service

8 providers in China[J]. AIDS Patient Care STDS, 2008, 22(6):513-520.

9 [4] China Ministry of Health and UN Theme Group on HIV/AIDS in China. A joint assessment of HIV/AIDS prevention treatment and care in China. Beijing China Ministry of Health Dec, 2003.

[5] Adams B. Locked doors: the human rights of people living with HIV / AIDS in China.. New York New York Human Rights Watch Sep, 2003(4):675-676.

[6] Peng X U, Chen W, Lahong J U, et al. Evolution and perfection of the HIV/AIDS designated hospital system in China. Chinese Journal of Health Policy, 2015 (5): 67-72.(in Chinese)

[7] Wagner, A. C., Hart, T. A., Mcshane, K. E., Margolese, S., \& Girard, T. A. Health care provider attitudes and beliefs about people living with HIV: Initial validation of the Health Care Provider HIV/AIDS Stigma Scale (HPASS). AIDS Behav, 2014, 18(12):2397-408.

[8] Stein J A, Li L. Measuring HIV-related Stigma Among Chinese Service Providers: Confirmatory Factor Analysis of a Multidimensional Scale. AIDS Behav, 2008, 12(5):789-795.

[9] Rutledge, S. E., Whyte, J., Abell, N., Brown, K. M., \& Cesnales, N. I. Measuring stigma among health care and social service providers: The HIV/AIDS Provider Stigma Inventory. Aids Patient Care \& Stds, 2011, 25(11):673.

[10]Windsor, L. C., Benoit, E., Ream, G. L., \& Forenza, B. The provider perception 
1 inventory: psychometrics of a scale designed to measure provider stigma about HIV,

2 substance abuse, and MSM behavior. Aids Care, 2013, 25(5):586-591.

3 [11]Ahmadi, K., Reidpath, D. D., Allotey, P., \& Hassali, M. A. A. A latent trait 4 approach to measuring HIV/AIDS related stigma in healthcare professionals: 5 application of mokken scaling technique. Bmc Medical Education, 2016, 16(1):155.

6 [12]Wang, L.; Ding, Z.; Qin, Q.; Cai, C.; Guo, W.; Cui, Y. Characteristics of HIV 7 transmission through heterosexual contact in China, 2008 - 2014. Zhonghua 8 Liuxingbingxue Zazhi 2015, 36, 1332 - 1336.

9 [13]Earnshaw V A, Chaudoir S R. From Conceptualizing to Measuring HIV Stigma:

A Review of HIV Stigma Mechanism Measures. AIDS Behav, 2009; 13(6):1160-1177.

[14]Tu Y S. Development of discrimination related HIV / AIDS scale for health work. Kunming Medical College, 2006.(in Chinese)

[15] Patterson D, London L. International law, human rights and HIV/AIDS. Bull

World Health Organ. 2002;80(12):964-9.

[16] Cao Y, Li X, Tao Y, et al. Development and evaluation of discrimination related HIV/AIDS scale for medical and nursing students. Chinese Journal of Disease Control \& Prevention, 2013, 12: 017.(in Chinese)

[17] Brown TA. Confirmatory factor analysis for applied research. First. New York:The Guilford Press; 2006.

[18] Polit D F, Beck C T. The content validity index: are you sure you know what's being reported? Critique and recommendations. Res Nurs Health, 2006, 29(5): 489-497.

[19] Ferguson E, Cox T. Exploratory factor analysis: A users' guide. International Journal of Selection and Assessment, 1993, 1(2): 84-94.

[20] Streiner D L, Norman G, Cairney J. Health Measurement Scales: A practical guide to their development and use. Oxford. UK: Oxford University Press, 2015:156. 
1 [21] Sousa V D, Rojjanasrirat W. Translation, adaptation and validation of instruments

2 or scales for use in cross- cultural health care research: a clear and user- friendly

3 guideline. Journal of Evaluation in Clinical Practice, 2015, 17(2):268-274.

4 [22] Lynn M R. Determination and quantification of content validity. Nursing 5 Research, 1986, 35(6):382-385.

6 [23] Fleiss J L, Cohen J. The equivalence of weighted kappa and the intraclass 7 correlation coefficient as measures of reliability. Educational and psychological 8 measurement, 1973, 33(3): 613-619.

9 [24]Beck C T, Gable R K. Ensuring content validity: An illustration of the process. Journal of Nursing measurement, 2001, 9(2): 201-215.

[25]Polit D F, Beck C T, Owen S V. Is the CVI an acceptable indicator of content validity? Appraisal and recommendations. Res Nurs Health, 2007, 30(4): 459-467.

[26] Landis J R, Koch G G. The measurement of observer agreement for categorical data. Biometrics, 1977, 33(1):159-174.

[27] Ding W, Song P X K. EM algorithm in Gaussian copula with missing data. Computational Statistics \& Data Analysis, 2016, 101: 1-11.

[28] Doka P J S, Danjin M, Dongs I S. HIV/AIDS-related stigma and discrimination among health-care providers in a tertiary health facility. Journal of Medical Sciences, 2017, 37(2): 44.

[29] Hibbert M, Wolton A, Crenna-Jennings W, et al. Experiences of stigma and discrimination in social and healthcare settings among trans people living with HIV in the UK. AIDS care, 2018, 30(7): 836-843.

[30] Stringer K L, Turan B, McCormick L, et al. HIV-related stigma among healthcare providers in the deep south. AIDS Behav, 2016, 20(1): 115-125.

[31] Magnus, M., Herwehe, J., Murtazarossini, M., et al. Linking and retaining HIV patients in care: the importance of provider attitudes and behaviors. AIDS Patient Care STDS, 2013, 27(5):297-303. 
1 [32] Stochl J, Jones P B, Croudace T J. Mokken scale analysis of mental health and

2 well-being questionnaire item responses: a non-parametric IRT method in empirical

3 research for applied health researchers. BMC Med Res Methodol, 2012, 12(1):1-16.

4 [33] Dean K, Walker Z, Jenkinson C. Data quality, floor and ceiling effects, and

5 test-retest reliability of the Mild Cognitive Impairment Questionnaire. Patient Relat

6 Outcome Meas, 2018, 9:43-47. 


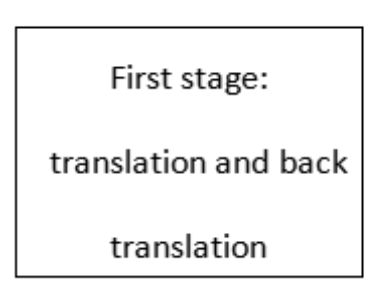

\begin{tabular}{|l|}
\hline Second stage: \\
content review \\
\hline
\end{tabular}
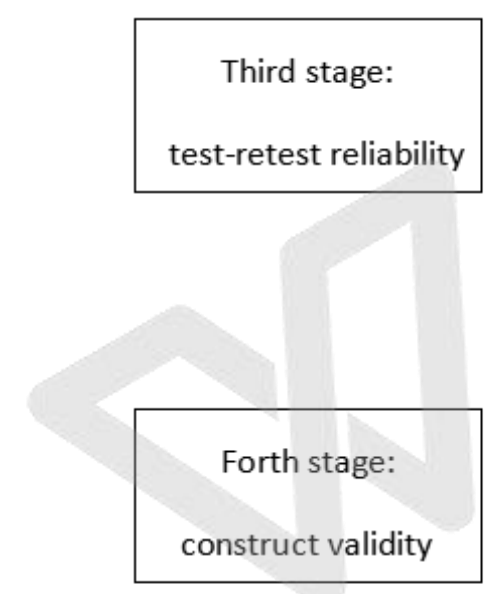

construct validity
The original Health Care Provider HIV/ AIDS Stigma

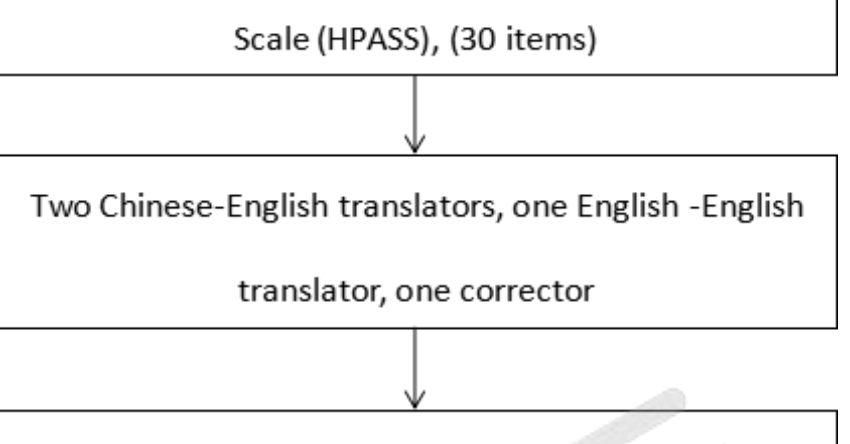

Six experts (2 psychiatrists, 4 infection specialists) rated Scale Content Validity S-CVI / Ave =0.44; I-CVI / Ave =

0.33-1.00, one item deleted, 29 items remained
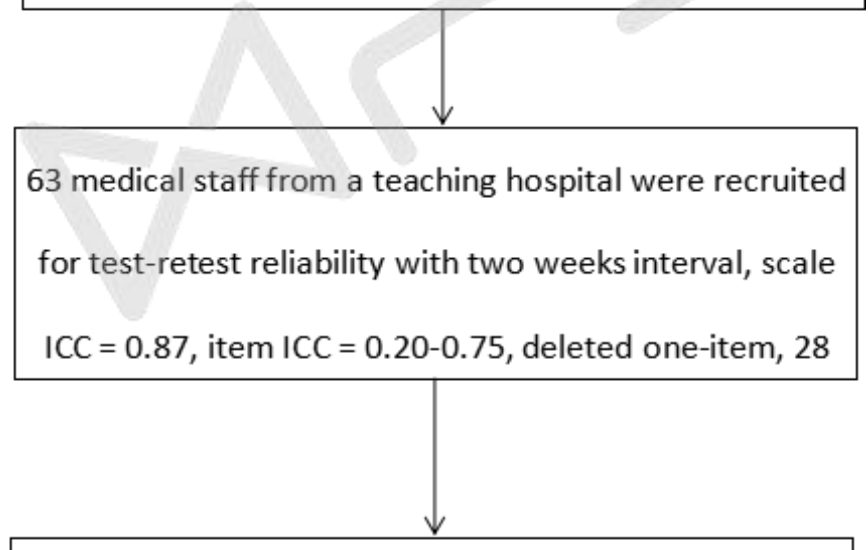

349 medical staff from 52 hospitals Sichuan province surveyed. Exploratory factor analysis resulted in 16 items with three factors of "discrimination", "prejudice" and "stereotypes", $\quad$ explained $59.01 \%$ variance. The Cronbach's $\alpha$ of the total scale was 0.88 , and $0.88,0.86$ and 0.74 for three factors respectively. 
Table 1. Demographics and HPASS score $(N=349)$

\begin{tabular}{|c|c|c|c|c|c|}
\hline Project & Frequency & Percentage & Mean (SD) & $t / F$ & $\mathbf{P}$ \\
\hline Age & & & $31.74(8.46)$ & & \\
\hline \multicolumn{6}{|l|}{ Gender } \\
\hline Male & 33 & 9.5 & $52.03(11.74)$ & $0.336^{t}$ & 0.737 \\
\hline Female & 316 & 90.5 & $51.28(12.35)$ & & \\
\hline \multicolumn{6}{|l|}{ Jobs } \\
\hline Clinical medical & 48 & 13.8 & \multirow{3}{*}{$\begin{array}{l}51.81(11.36) \\
51.23(12.51)\end{array}$} & \multirow[t]{3}{*}{$0.301^{t}$} & \multirow[t]{3}{*}{0.763} \\
\hline Nursing & 285 & 81.7 & & & \\
\hline Other & 16 & 4.5 & & & \\
\hline \multicolumn{6}{|l|}{ Education } \\
\hline Master graduate and above & 50 & 14.3 & $50.48(11.65)$ & \multirow[t]{3}{*}{$0.690^{F}$} & \multirow[t]{3}{*}{0.502} \\
\hline Undergraduate & 183 & 52.4 & $52.08(11.61)$ & & \\
\hline College and below & 116 & 33.2 & $50.56(13.53)$ & & \\
\hline \multicolumn{6}{|l|}{ Title of medical staff } \\
\hline Senior & 48 & 13.8 & $46.50(9.97)$ & \multirow[t]{3}{*}{$3.137^{F}$} & \multirow[t]{3}{*}{0.026} \\
\hline Intermediate & 61 & 17.5 & $51.77(11.26)$ & & \\
\hline Primary & 240 & 68.8 & $52.08(12.75)$ & & \\
\hline \multicolumn{6}{|l|}{ Department } \\
\hline Infectious Diseases & 116 & 33.2 & $47.91(11.24)$ & $3.755^{t}$ & 0.001 \\
\hline
\end{tabular}


Have you ever worked with HIV/AIDS?

Yes

No

$\mathrm{t}=$ Independent Sample $\mathrm{t}$-test $; \mathrm{F}=$ One-Way ANOVA
331

18
54.39 (14.39) 
Table 2. Eigen values and explanatory variables, Mean (SD) and Factor correlation analysis of the scale

\begin{tabular}{ccccc}
\hline Factor & Eigenvalue & Variance Percent & Cumulative Percentage & Mean (SD) \\
\hline Discrimination & 6.06 & 37.85 & 37.85 & $2.39(0.96)$ \\
Prejudice & 2.00 & 12.50 & 50.35 & $3.47(0.96)$ \\
Stereotypes & 1.48 & 9.26 & 59.61 & $3.74(0.83)$ \\
\hline
\end{tabular}


Table 3. Factor correlation analysis of the scale

\begin{tabular}{|c|c|c|c|}
\hline Factor & Discrimination & Prejudice & Stereotypes \\
\hline Discrimination & 1 & & \\
\hline Prejudice & $.602^{\mathrm{c}}$ & 1 & \\
\hline Stereotypes & $.342^{\mathrm{c}}$ & $.529^{c}$ & 1 \\
\hline
\end{tabular}

${ }^{\mathrm{c}}$ Notes: $\mathrm{P}<0.01$ 
Table 4. Factor analysis of the scale, Internal consistency, Compare Mean (SD) and Ceiling and floor effect

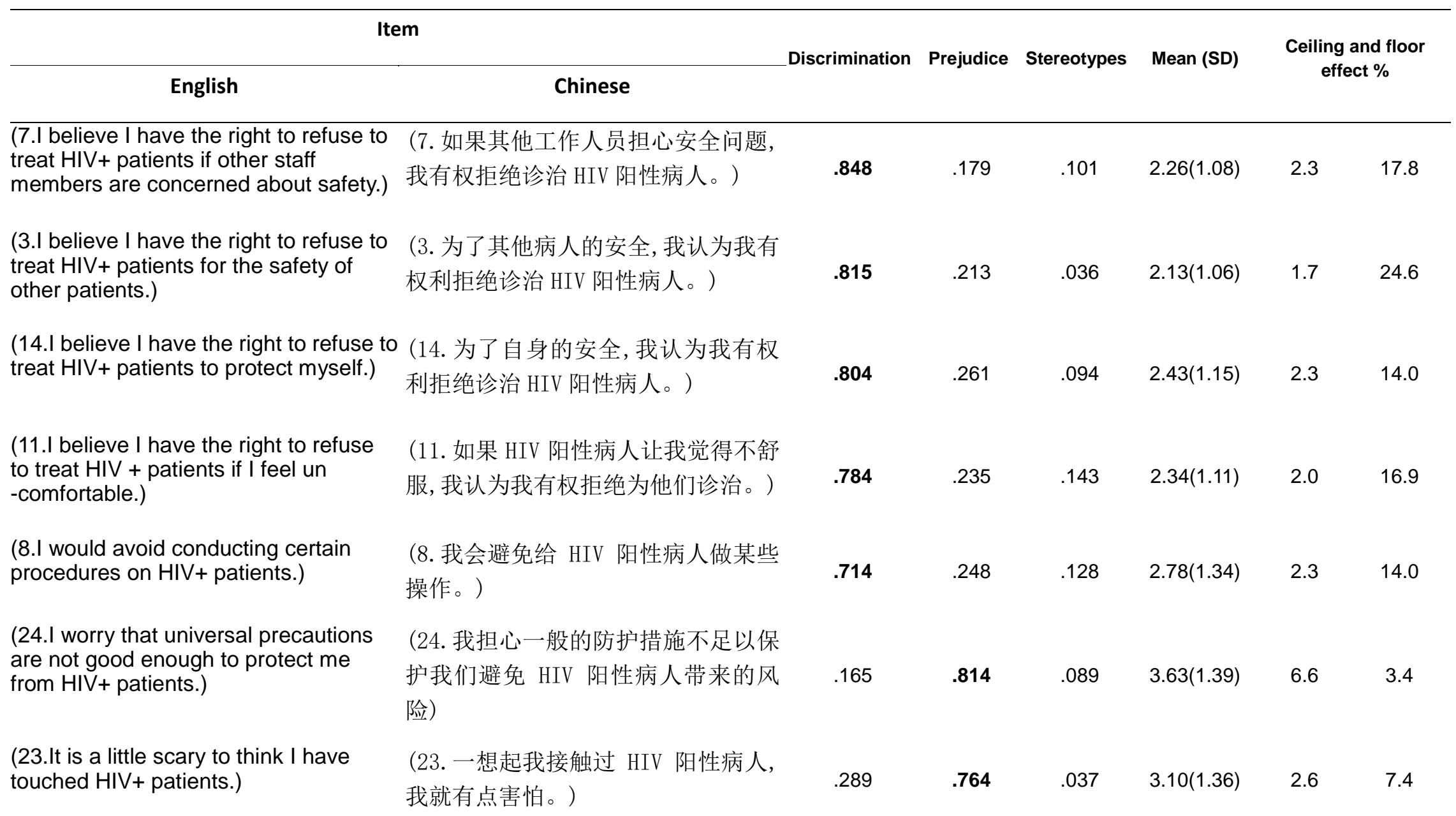


(19.I worry about contracting HIV from HIV + patients.)

(25.I would feel uncomfortable knowing one of my colleagues is $\mathrm{HIV}+$.)

(21. HIV + patients make me uncomfortable.)

(29.It would be hard to react calmly if a patient tells me he or she is HIV+.)

(10.HIV + patients tend to have numerous sexual partners.)

(9.I think if people act responsibly they will not contract HIV.)

(4.I think people would not get HIV if they had sex with fewer people.)

(1.I believe most HIV +patients
acquired the virus through risky acquired the
behaviour.)

(20.I often think HIV + patients have caused their own health problems.)
（19. 我担心从 HIV 阳性病人那感染 HIV。)

（25. 知道一个同事是 HIV 阳性, 这会 让我在和他相处时感到不舒服。）

（21. HIV 阳性的病人让我感到不舒 服。）

.318

(29. 如果一个病人告诉我他/她是 HIV 阳性, 那我很难平静地做出回 应。)

（10. HIV 阳性病人往往有很多性伴。）

073

(9. 我认为如果人们有一点的责任感, 他们就不会感染 HIV。)

(4. 我认为如果人们不乱搞男女关系, 他们就不会感染 HIV。）

(1. 我相信大多数的 HIV 阳性病人是 通过高危性行为感染 HIV 的。)

(20. 我通常认为 HIV 阳性病人的健康 问题是自己造成的。)

.090
.722

.127

$4.27(1.31)$

13.2

.708

.219

3.50(1.32)

4.9

.625

.327

3.38(1.30)

4.0

.609

.147

3.01(1.31)

.107

.740

3.69(1.34)

6.9

.060

.142

.725

3.58(1.42)
.89

.86

Notes: The item 19 has one values missing(the missing rate was $0.28 \%$ ) 
